\title{
Amphetamine-like Neurochemical and Cardiovascular Effects of $\alpha$-Ethylphenethylamine Analogs Found in Dietary Supplements
}

\author{
Charles W. Schindler, Eric B. Thorndike, John S. Partilla, Kenner C. Rice, \\ and Michael H. Baumann
}

\begin{abstract}
Designer Drug Research Unit (C.W.S., J.S.P., M.H.B.) and Preclinical Pharmacology Section (C.W.S., E.B.T.), National Institute on Drug Abuse Intramural Research Program, Baltimore, Maryland and Drug Design and Synthesis Section, National Institute on Drug Abuse and National Institute of Alcohol Abuse and Alcoholism Intramural Research Programs, Rockville, Maryland (K.C.R.)
\end{abstract}

Received May 26, 2020; accepted October 16, 2020

\begin{abstract}
Dietary supplements often contain additives not listed on the label, including $\alpha$-ethyl homologs of amphetamine such as $\mathrm{N}, \alpha$ diethylphenethylamine (DEPEA). Here, we examined the neurochemical and cardiovascular effects of $\alpha$-ethylphenethylamine (AEPEA), $N$-methyl- $\alpha$-ethylphenethylamine (MEPEA), and DEPEA as compared with the effects of amphetamine. All drugs were tested in vitro using uptake inhibition and release assays for monoamine transporters. As expected, amphetamine acted as a potent and efficacious releasing agent at dopamine transporters (DAT) and norepinephrine transporters (NET) in vitro. AEPEA and MEPEA were also releasers at catecholamine transporters, with greater potency at NET than DAT. DEPEA displayed fully efficacious release at NET but weak partial release at DAT (i.e., $40 \%$ of maximal effect). In freely moving, conscious male rats fitted with biotelemetry transmitters for physiologic monitoring, amphetamine (0.1-3.0 $\mathrm{mg} / \mathrm{kg}$, s.c.) produced robust dose-related increases in blood pressure (BP), heart rate (HR),
\end{abstract}

and motor activity. AEPEA (1-10 $\mathrm{mg} / \mathrm{kg}$, s.c.) produced significant increases in BP but not HR or activity, whereas DEPEA and MEPEA (1-10 mg/kg, s.c.) increased BP, $H R$, and activity. In general, the phenethylamine analogs were approximately 10 -fold less potent than amphetamine. Our results show that $\alpha$-ethylphenethylamine analogs are biologically active. Although less potent than amphetamine, they produce cardiovascular effects that could pose risks to humans. Given that MEPEA and DEPEA increased locomotor activity, these substances may also have significant abuse potential.

\section{SIGNIFICANCE STATEMENT}

The $\alpha$-ethyl homologs of amphetamine have significant cardiovascular, behavioral, and neurochemical effects in rats. Given that these compounds are often not listed on the ingredient labels of dietary supplements, these compounds could pose a risk to humans using these products.

\section{Introduction}

Nutritional supplements often contain ingredients that are not listed on the product labels, including analogs of phenethylamine (PEA) that display structural similarity to amphetamine (Eichner, 2014; Pawar and Grundel, 2017). We recently showed that the supplement additive $\beta$-methylphenethylamine (BMPEA), a positional isomer of amphetamine (i.e., $\alpha$-methylphenethylamine), increases blood pressure (BP) in rats and may therefore produce adverse effects in humans (Schindler et al., 2019). Another PEA analog previously found in dietary supplements is $N, \alpha$-diethylphenethylamine (DEPEA; see Fig. 1 for chemical structure). DEPEA has been detected in powdered material confiscated for drug trafficking (Lee et al., 2013) and in supplement products destined for human consumption

This research was supported by the Intramural Research Program of the National Institutes of Health National Institute on Drug Abuse and the National Institute of Alcohol Abuse and Alcoholism grants [Z01DA000523, Z01DA000532]

https://doi.org/10.1124/jpet.120.000129.
(ElSohly and Gul, 2014; Cohen et al., 2014; Wahlstrom et al., 2014; ElSohly et al., 2015).

Urine toxicology testing has also confirmed the presence of DEPEA in users of some dietary supplements (Wójtowicz et al., 2015). For example, in urine samples obtained through routine toxicological testing, DEPEA was detected in samples from individuals who used a dietary supplement suspected of containing added DEPEA (Uralets et al., 2014). In addition to DEPEA, $\alpha$-ethylphenethylamine (AEPEA) was also found in some of the same samples, potentially the result of $N$-dealkylation of DEPEA via hepatic metabolism. Both DEPEA and AEPEA have been found in urine samples collected by the World Anti-Doping Agency, demonstrating exposure to these substances among athletes (World AntiDoping Agency, 2012, 2013). In a survey of German athletes who were asked about their supplement use, some respondents specifically reported seeking products containing DEPEA (Dreher et al., 2018). Since DEPEA and related analogs are not listed as ingredients on the labels of supplement products, the FDA considers them adulterants and requires companies

ABBREVIATIONS: AEPEA, $\alpha$-ethylphenethylamine; BMPEA, $\beta$-methylphenethylamine; BP, blood pressure; Cl, confidence interval; DAT, dopamine transporter; DEPEA, $N, \alpha$-diethylphenethylamine; Emax, maximal release; FEDA, Food and Drug Administration; GPCR, G protein-coupled receptor; HR, heart rate; 5-HT, serotonin; IRP, Intramural Research Program; MEPEA, N-methyl- $\alpha$-ethylphenethylamine; MPP ${ }^{+}$, methyl-4-phenylpyridinium; NET, norepinephrine transporter; NIDA, National Institute on Drug Abuse; PEA, phenethylamine; SERT, serotonin transporter. 
<smiles>CC(N)Cc1ccccc1</smiles>

amphetamine<smiles>CNC(C)Cc1ccccc1</smiles>

methamphetamine<smiles>CCC(N)Cc1ccccc1</smiles>

$\alpha$-ethyl-PEA (AEPEA)<smiles>CCC(Cc1ccccc1)NC</smiles>

$N$-methyl- $\alpha$-ethyl-PEA (MEPEA)<smiles>CCNC(CC)Cc1ccccc1</smiles>

$N, \alpha$-diethyl-PEA (DEPEA)
Fig. 1. Chemical structures of AEPEA, MEPEA, and DEPEA as compared with amphetamine and methamphetamine. selling the products to remove them from the market (Pawar and Grundel, 2017). However, PEA analogs may still be present in those supplements not tested by the FDA.

Despite the apparent widespread use of DEPEA and similar compounds, very little is known about their biologic effects. Oberlender and Nichols (1991) reported that AEPEA partially generalizes to the discriminative stimulus effects of amphetamine. In that study, rats were trained to respond on one lever when injected with amphetamine and another lever when injected with saline. When the trained rats received a noncontingent injection of AEPEA, they responded on the amphetamine-associated lever, suggesting that AEPEA and amphetamine might share common effects. Santillo (2014) reported that AEPEA inhibits human monoamine-oxidase type $\mathrm{A}$ in vitro in a competitive and reversible manner similar to amphetamine, whereas DEPEA has much weaker effects in this regard. Liu and Santillo (2016) reported that DEPEA inhibits activity of the hepatic cytochrome P450 enzyme CYP2D6, which could alter the effects of other drugs taken in combination with DEPEA. Because of the paucity of information about the pharmacology of $\alpha$-ethyl PEA analogs, we sought to study the effects of AEPEA and its amine-substituted analogs, $N$-methyl- $\alpha$-ethylphenethylamine (MEPEA) and DEPEA, as compared with the effects of amphetamine (see Fig. 1 for chemical structures). Based on their structural similarities to amphetamine, we expected that these PEA analogs would have similar effects to amphetamine, although at potentially different potencies.

\section{Materials and Methods}

Drugs and Reagents. AEPEA, MEPEA, and DEPEA were synthesized using standard organic chemical reactions and techniques as follows: AEPEA was prepared by reductive amination of 1phenyl-2-butanone (TCI America Research Chemicals, Portland, OR) using the method of González-Sabin et al. (2002). The distilled base was then converted to the $\mathrm{HCl}$ salt in acetonitrile-ether. MEPEA was prepared in two steps by first $N$-formylation of AEPEA with ethyl formate at $135^{\circ} \mathrm{C}$ for 18 hours in a pressure bottle. The resulting $N$-formyl derivative was distilled and then reduced to MEPEA with Vitride in refluxing Toluene. The distilled base was then converted to the oxalate salt in acetonitrile. DEPEA was synthesized in two steps by $N$-acetylation of AEPEA with acetic anhydride in a chloroformsaturated sodium bicarbonate two-phase system, followed by reduction of the resulting $\mathrm{N}$-acetyl derivative with lithium aluminum hydride in tetrahydrofuran. The distilled base was converted to the $\mathrm{HCl}$ salt in acetone-ether. Each compound was fully characterized with appropriate high-resolution mass spectral and 400-MHz NMR analyses and with appropriate combustion analyses for carbon, hydrogen, and nitrogen. Each compound was chromatographically homogenous by thin-layer chromatography. Chemical purity for each compound was estimated to be greater than $98 \%$. The corresponding $\alpha$-methyl comparator compound ( $S$ )-amphetamine sulfate (amphetamine) was obtained from the pharmacy at the National Institute on Drug Abuse (NIDA), Intramural Research Program (IRP), in Baltimore, MD. $\left[{ }^{3} \mathrm{H}\right]$ Methyl-4-phenylpyridinium $\left(\left[{ }^{3} \mathrm{H}\right] \mathrm{MPP}^{+} ; 80 \mathrm{Ci} / \mathrm{mmol}\right)$ was purchased from American Radiolabeled Chemicals (St. Louis, $\mathrm{MO}$ ), and ${ }^{3} \mathrm{H}$-neurotransmitters $(30-50 \mathrm{Ci} / \mathrm{mmol})$ were purchased from Perkin Elmer (Shelton, CT). All other chemicals and reagents were acquired from Sigma-Aldrich (St Louis, MO) unless otherwise noted. Drugs for the in vivo experiments were dissolved in sterile saline, and doses are expressed as the salts.

Animals. Male Sprague-Dawley rats were used for all experiments, as described in detail below. All procedures were approved by the Animal Care and Use Committee of the NIDA IRP and were carried out in accordance with the Guide for the Care and Use of Laboratory Animals. Animals were housed in facilities fully accredited by the Association for the Assessment and Accreditation of Laboratory Animal Care.

In Vitro Transporter Assays. In total, 28 male Sprague-Dawley rats (Charles River, Kingston, NY) weighing 250-300 g were used for the synaptosome assays. Rats were group-housed with free access to food and water under a 12-hour light/dark cycle with lights on at 0700 hours. Rats were euthanized by $\mathrm{CO}_{2}$ narcosis, and synaptosomes were prepared from brains using standard procedures (Rothman et al., 2003). Transporter uptake and release assays were performed as described previously (Solis et al., 2017). In brief, synaptosomes were prepared from caudate tissue for dopamine transporter (DAT) assays and from whole brain minus caudate and cerebellum for norepinephrine transporter (NET) and serotonin (5-HT) transporter (SERT) assays.

For uptake inhibition assays, $5 \mathrm{nM}\left[{ }^{3} \mathrm{H}\right]$ dopamine, $\left[{ }^{3} \mathrm{H}\right]$ norepinephrine, or $\left[{ }^{3} \mathrm{H}\right] 5-\mathrm{HT}$ was used for DAT, NET, or SERT assays, respectively. To optimize uptake for a single transporter, unlabeled blockers were included to prevent the uptake of ${ }^{3} \mathrm{H}$-transmitter by competing transporters. Uptake inhibition was initiated by incubating synaptosomes with various doses of test compound and ${ }^{3} \mathrm{H}$-transmitter in Krebs-phosphate buffer. Uptake assays were terminated by rapid vacuum filtration, and retained radioactivity was quantified with liquid scintillation counting (Baumann et al., 2013).

For release assays, $9 \mathrm{nM}\left[{ }^{3} \mathrm{H}\right] \mathrm{MPP}+$ was used as the radiolabeled substrate for DAT and NET, whereas $5 \mathrm{nM}\left[{ }^{3} \mathrm{H}\right] 5-\mathrm{HT}$ was used for SERT. All buffers used in the release assay contained $1 \mu \mathrm{M}$ reserpine to block vesicular uptake of substrates. The selectivity of release 
assays was optimized for a single transporter by including unlabeled blockers to prevent the uptake of $\left[{ }^{3} \mathrm{H}\right] \mathrm{MPP}+$ or $\left[{ }^{3} \mathrm{H}\right] 5$-HT by competing transporters. Synaptosomes were preloaded with radiolabeled substrate in Krebs-phosphate buffer for 1 hour to reach steady state. Release assays were initiated by incubating preloaded synaptosomes with various concentrations of the test drug. Release was terminated by vacuum filtration, and retained radioactivity was quantified by liquid scintillation counting. For substrate reversal experiments, the effects of AEPEA and MEPEA on transporter-mediated release were examined as described above in the presence or absence of $1 \mathrm{nM} 1$-[2[bis(4-fluorophenyl)methoxy]ethyl]-4-(3-phenylpropyl)piperazine (GBR12909) for DAT assays or $8 \mathrm{nM}$ desipramine for NET assays. Effects of test drugs on release were expressed as a percentage of maximal release, with maximal release (i.e., $100 \% \mathrm{E}_{\max }$ ) defined as the release produced by tyramine at doses that evoke the efflux of all "releasable" tritium by synaptosomes (10 $\mu \mathrm{M}$ tyramine for DAT and NET assay conditions, and $100 \mu \mathrm{M}$ tyramine for SERT assay conditions).

Effects of test drugs on uptake inhibition and release were analyzed by nonlinear regression using GraphPad Prism 8 (GraphPad Scientific, San Diego, CA). Dose-response values for the uptake inhibition and release were fit to the equation $\mathrm{Y}(x)=\mathrm{Y}_{\min }+\left(\mathrm{Y}_{\max }-\mathrm{Y}_{\min }\right) /(1+$ $\left.10 \exp \left[\left(\log P_{50}-\log x\right)\right] \times n\right)$, where $x$ is the concentration of the compound tested, $\mathrm{Y}(x)$ is the response measured, $\mathrm{Y}_{\max }$ is the maximal response, $\mathrm{P}_{50}$ is either $\mathrm{IC}_{50}$ (the concentration that yields halfmaximal uptake inhibition response) or $\mathrm{EC}_{50}$ (the concentration that yields half-maximal release), and $n$ is the Hill slope parameter. We employed the "EC ${ }_{50}$ shift test" in Prism to statistically evaluate the possibility of significant differences in potency across the drugs. Briefly, the $\mathrm{EC}_{50}$ shift compares dose-response curves for a series of drugs and can be used to test the null hypothesis that all drugs are equipotent (i.e., the $\mathrm{EC}_{50}$ ratio equals 1 ). When significant differences in drug potency are observed, the null hypothesis is rejected, and supporting $\mathrm{F}$ scores and $P$ values are given.

Receptorome Screening. AEPEA, MEPEA, DEPEA, and amphetamine were submitted to the psychoactive drug screening program program of the National Institute on Mental Health and evaluated for binding affinity at a variety of human $\mathrm{G}$ protein-coupled receptors (GPCRs) according to established protocols (Besnard et al., 2012; https://pdsp.unc.edu/pdspweb/content/UNC-CH\%20Protocol\% 20Book.pdf). Compounds were first screened at a fixed concentration of $10 \mu \mathrm{M}$ to assess inhibition of receptor binding. In those instances in which binding was inhibited by more than $50 \%$ at $10 \mu \mathrm{M}$, full doseeffect functions were obtained, and $\mathrm{K}_{\mathrm{i}}$ values were calculated by nonlinear regression using the Cheng-Prusoff equation.

In Vivo Biotelemetry. Five adult male Sprague-Dawley rats (Charles River, Kingston, NY) were used as subjects for the telemetry experiments. Rats were purchased by Data Sciences International (St. Paul, MN) and received surgically implanted HD-S10 biotelemetry transmitters. For the surgery, the rats were anesthetized with isoflurane and the abdominal cavity was opened. The descending aorta was isolated, and the catheter from the transmitter was inserted into the aorta and glued in place. The abdominal muscles and skin were then sutured to close the incision. Rats were treated with subcutaneous meloxicam after surgery. After recovery at Data Sciences International, the rats were shipped to the NIDA IRP in Baltimore, MD, and underwent a 7-day quarantine period.

After release from quarantine, the rats were individually housed in a temperature-controlled $\left(22.2 \pm 1.1^{\circ} \mathrm{C}\right)$ and humidity-controlled $(45 \% \pm 10 \%)$ vivarium on a 12 -hour reverse light/dark cycle (lights off at 0700) with free access to water. Food was restricted to maintain a constant or slowly increasing weight of approximately $400-500 \mathrm{~g}$ over the course of the experiment. The rats were subsequently adapted to the experimental chambers and injection procedure over a period of 3 to 4 weeks. Each weekday, rats were transported from the vivarium to a testing room where the food and water were removed from the home cage and the entire home cage was placed on top of a telemetry receiver (RPC-001; Data Sciences) inside a small acoustical chamber (BRS/LVE, Laurel, MD). Transmitters were turned on by placing a magnet near the abdomen of the rat. The chambers were then closed, and experimental parameters were monitored for 3 hours. At the end of the session, the transmitters were turned off by again placing a magnet near the abdomen of the rats, water and food were returned to the home cages, and the rats were returned to the vivarium housing room. Once experimental parameters were stable from day to day, injections of saline were given subcutaneously twice per week (typically on Tuesdays and Fridays) 5 minutes prior to the rats being placed in the experimental chamber. Once experimental parameters were again stable after saline injections, drug or saline injection procedures began.

Dose-effect determinations for AEPEA (1-10 mg/kg), MEPEA (1$10 \mathrm{mg} / \mathrm{kg})$, DEPEA (1-10 mg/kg), and amphetamine $(0.1-3 \mathrm{mg} / \mathrm{kg})$ were determined first. Drugs were tested no more frequently than twice per week, typically Tuesday and Friday. Order for the dose-effect testing was nonsystematic, although all rats were typically tested with the same drug and dose on any given test day to simplify drug preparation and administration. All drugs were administered subcutaneously 5 minutes prior to placement of the cages in the experimental chambers. Saline was tested every 2 to 3 weeks, and responses after saline were stable over the testing period. Rats were 2-9 months of age over the course of the experiment. Rats for this study had been previously exposed to BMPEA, prazosin, and chlorisondamine but were allowed at least 3 weeks of washout prior to testing (Schindler et al., 2019).

Data from the transmitters were polled for 10 seconds every minute, and these 1-minute readings were used to construct time course profiles and calculate mean effects over the 3-hour session for statistical analysis. The transmitters supplied readings for BP, heart rate (HR, derived from the $\mathrm{BP}$ signal), core body temperature, and motor activity. Activity was measured continuously by tracking the strength of the transmitter radio signal as the rat moved about its home cage, but this measure does not have any units. Data for AEPEA time course were subject to a mixed-effects analysis, whereas the data for each drug and measurement in the dose-effect study were subject to ANOVA with follow-up tests using the Dunnett's multiple comparison test, which can compare drug effects to control (GraphPad Prism, version 8). $\mathrm{ED}_{50}$ values for the effects of drugs on $\mathrm{BP}$ were calculated using nonlinear regression, and these potency values were compared using the $\mathrm{EC}_{50}$ shift test in Prism.

\section{Results}

Transporter Uptake and Release Assays. Figure 2 depicts the dose-response curves for inhibition of ${ }^{3} \mathrm{H}$-neurotransmitter uptake and stimulation of $\left[{ }^{3} \mathrm{H}\right] \mathrm{MPP}^{+}$efflux (i.e., release) at DAT and NET. None of the compounds showed measurable ability to inhibit uptake or stimulate release at SERT for doses up to $10 \mu \mathrm{M}$ (data not shown). Table 1 summarizes the $\mathrm{IC}_{50}$ values for uptake inhibition and the $\mathrm{EC}_{50}$ and $\% \mathrm{E}_{\max }$ values for release at DAT and NET. Amphetamine was the most potent uptake inhibitor at DAT $\left(\mathrm{IC}_{50}=122 \mathrm{nM}\right)$ and NET $\left(\mathrm{IC}_{50}=69 \mathrm{nM}\right)$. DEPEA was 5 -fold less potent than amphetamine at DAT, whereas the other compounds were even weaker at DAT, with $\mathrm{IC}_{50}$ values $>1 \mu \mathrm{M}$. $\mathrm{EC}_{50}$ shift analysis showed that amphetamine was significantly more potent at DAT inhibition than all PEA analogs $\left(\mathrm{F}_{3,89}=578\right.$, $P<0.0001$ ), whereas DEPEA was more potent than AEPEA and MEPEA in this regard $\left(\mathrm{F}_{2,66}=256, P<0.0001\right)$. All of the PEA analogs had $\mathrm{IC}_{50}$ values for NET inhibition that were 6 - to 8-fold less potent than amphetamine. $\mathrm{EC}_{50}$ shift analysis demonstrated that amphetamine was significantly more potent at NET inhibition than all PEA analogs 

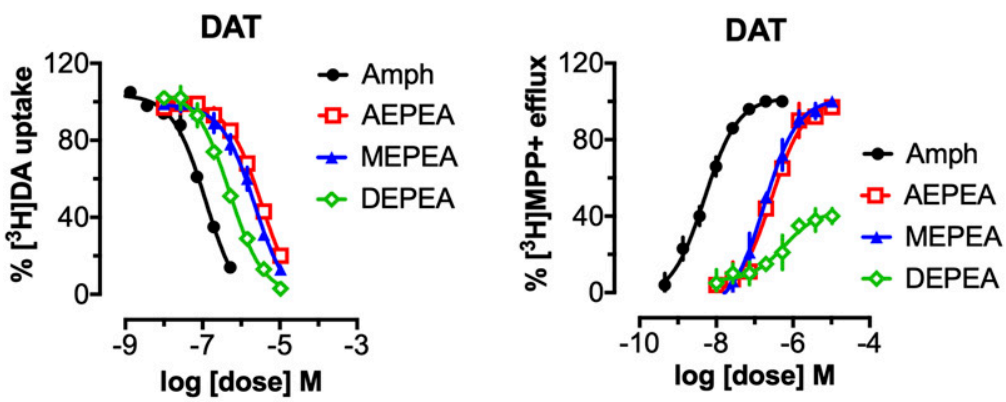

Fig. 2. Effects of amphetamine and PEA analogs on inhibition of uptake and stimulation of efflux (i.e., release) at DAT (upper panels) or NET (lower panels) in rat brain synaptosomes. For uptake assays, synaptosomes were incubated with different concentrations of test drugs in the presence of $5 \mathrm{nM}\left[{ }^{3} \mathrm{H}\right]$ dopamine $\left(\left[{ }^{3} \mathrm{H}\right] \mathrm{DA}\right)$ or $\left[{ }^{3} \mathrm{H}\right]$ norepinephrine $\left(\left[{ }^{3} \mathrm{H}\right] \mathrm{NE}\right)$. Data are means \pm S.D., expressed as a percentage of transmitter uptake for $N=3$ experiments performed in triplicate. For release assays, synaptosomes were preloaded with $9 \mathrm{nM}\left[{ }^{3} \mathrm{H}\right] \mathrm{MPP}^{+}$and then incubated with different concentrations of test drugs to evoke release via reverse transport. Data are means \pm S.D., expressed as a percentage of $\left[{ }^{3} \mathrm{H}\right] \mathrm{MPP}^{+}$release for three

experiments performed in triplicate.
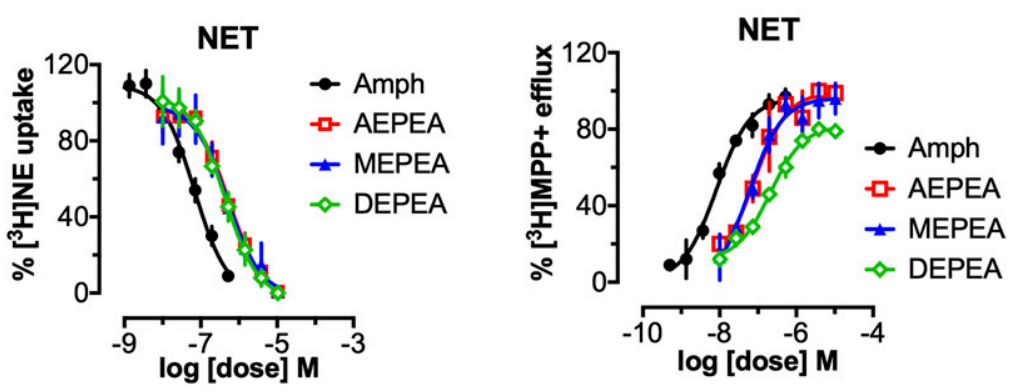

$\left(\mathrm{F}_{3,89}=62.7, P<0.0001\right)$, but potencies for AEPEA, MEPEA, and DEPEA to inhibit NET did not differ from each other.

As expected, amphetamine was a fully efficacious substratetype releasing agent with high potency at $\mathrm{DAT}\left(\mathrm{EC}_{50}=5 \mathrm{nM}\right)$ and NET $\left(\mathrm{EC}_{50}=8 \mathrm{nM}\right)$. AEPEA and MEPEA were much less potent than amphetamine as releasers at DAT but were approximately equipotent with each other at this transporter. It is noteworthy that DEPEA was a weak partial releaser at DAT, achieving only $40 \%$ of the maximal release response (i.e., partial agonist effect). $\mathrm{EC}_{50}$ shift analysis showed that amphetamine was significantly more potent as a releaser at DAT when compared with PEA compounds $\left(\mathrm{F}_{3,89}=374\right.$, $P<0.0001$ ), whereas DEPEA was significantly less potent than AEPEA and MEPEA in this regard $\left(\mathrm{F}_{2,57}=158\right.$, $P<0.0001)$. All of the PEA compounds were efficacious substrate-type releasers at NET. EC $_{50}$ shift analysis demonstrated that amphetamine was a more potent releaser at NET when compared with PEA compounds $\left(\mathrm{F}_{3,89}=96.2, P<0.0001\right)$, and DEPEA was less potent than AEPEA and $\operatorname{MEPEA}\left(\mathrm{F}_{2,66}=\right.$ 29.6, $P<0.0001$ ). In general, AEPEA and MEPEA displayed greater potency as releasers at $\mathrm{NET}\left(\mathrm{EC}_{50}=80\right.$ and $\left.58 \mathrm{nM}\right)$ when compared with DAT $\left(\mathrm{EC}_{50}=273\right.$ and $\left.179 \mathrm{nM}\right)$.

To explore the mechanism of ${ }^{3} \mathrm{H}$-neurotransmitter efflux produced by the efficacious releasers AEPEA and MEPEA, we carried out "substrate reversal" studies. These experiments involved testing the ability of selective uptake blockers to antagonize the releasing actions of the drugs. Figure 3 illustrates the effects of the selective DAT uptake inhibitor GBR12909 (1 nM) or the selective NET uptake inhibitor desipramine $(8 \mathrm{nM})$ on releasing activity produced by AEPEA and MEPEA. The results show that GBR12909 produced a parallel rightward shift in the DAT release curves for AEPEA and MEPEA, suggesting that both drugs interact at the orthosteric site on DAT to elicit their releasing actions. An $\mathrm{EC}_{50}$ shift analysis confirmed that GBR12909 significantly reduced the potency of AEPEA $\left(\mathrm{F}_{1,43}=1875, P<0.0001\right)$ and $\operatorname{MEPEA}\left(\mathrm{F}_{1,43}=504, P<0.0001\right)$ at DAT. For the NET substrate reversal experiments, desipramine shifted the NET release curves for AEPEA and MEPEA to the right, demonstrating that these drugs interact at NET sites. The $\mathrm{EC}_{50}$ shift results confirmed that desipramine significantly reduced the potency of AEPEA $\left(\mathrm{F}_{1,43}=64, P<0.0001\right)$ and $\operatorname{MEPEA}\left(\mathrm{F}_{1,43}=\right.$ $109, P<0.0001)$ at NET.

Receptorome Screening. Table 2 presents the results for AEPEA, MEPEA, and DEPEA in the human GPCR screening in comparison with amphetamine. In general, the PEA analogs had little activity at GPCRs when tested at a $10 \mu \mathrm{M}$ concentration (see receptors listed in footnote of Table 2).

TABLE 1

Effects of amphetamine and PEA analogs on the uptake of $\left[{ }^{3} \mathrm{H}\right]$ neurotransmitters and on the release of $\left[{ }^{3} \mathrm{H}\right] \mathrm{MPP}+$ at DAT or NET in rat brain synaptosomes

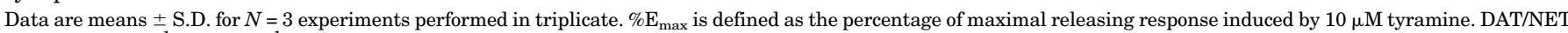
ratio $=\left(\mathrm{DAT} \mathrm{IC}_{50}^{-1}\right) /\left(\mathrm{NET} \mathrm{IC}_{50}^{-1}\right)$; higher value indicates greater DAT selectivity.

\begin{tabular}{|c|c|c|c|}
\hline Drug & Uptake Inhibition at DAT $\left[\mathrm{IC}_{50}(\mathrm{nM})\right]$ & Uptake Inhibition at NET $\left[\mathrm{IC}_{50}(\mathrm{nM})\right]$ & DAT/NET Ratio \\
\hline Amphetamine & $122 \pm 12$ & $69 \pm 9$ & 0.56 \\
\hline AEPEA & $3366 \pm 333$ & $573 \pm 78$ & 0.16 \\
\hline MEPEA & $2248 \pm 245$ & $503 \pm 95$ & 0.22 \\
\hline DEPEA & $510 \pm 38$ & $427 \pm 60$ & 0.84 \\
\hline & Release at DAT $\mathrm{EC}_{50}(\mathrm{nM})\left(\% \mathrm{E}_{\max }\right)$ & Release at NET $\mathrm{EC}_{50}(\mathrm{nM})\left(\% \mathrm{E}_{\max }\right)$ & \\
\hline Amphetamine & $5 \pm 1(102)$ & $8 \pm 1(96)$ & 1.6 \\
\hline AEPEA & $273 \pm 36(101)$ & $80 \pm 17(100)$ & 0.29 \\
\hline MEPEA & $179 \pm 25(101)$ & $58 \pm 12(96)$ & 0.32 \\
\hline DEPEA & $604 \pm 159(43)$ & $209 \pm 35(82)$ & 0.35 \\
\hline
\end{tabular}



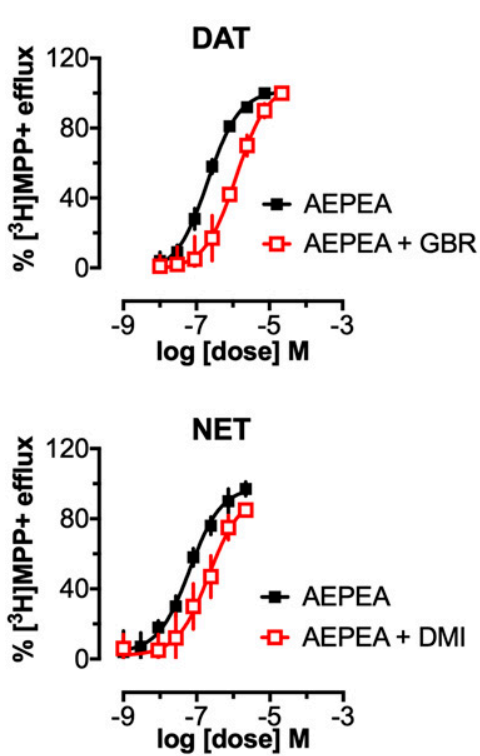
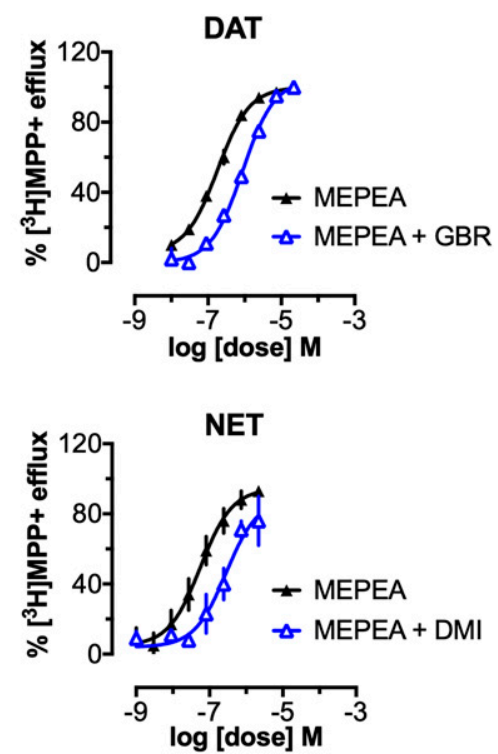

Fig. 3. Effects of GBR12909 (GBR) or desipramine (DMI) on drug-induced release of $\left[{ }^{3} \mathrm{H}\right] \mathrm{MPP}^{+}$at DAT (upper panels) or NET (lower panels). For substrate reversal assays, synaptosomes were preloaded with $9 \mathrm{nM}\left[{ }^{3} \mathrm{H}\right] \mathrm{MPP}^{+}$, and then test drugs were incubated with or without GBR12909 (1 nM) for DAT assays or desipramine ( $8 \mathrm{nM})$ for the NET assays. Data are means \pm S.D. expressed as a percentage of $\left[{ }^{3} \mathrm{H}\right] \mathrm{MPP}^{+}$ release for three experiments performed in triplicate.
Specific exceptions included the $5-\mathrm{HT}_{1 \mathrm{~A}}$ receptor, in which MEPEA and DEPEA had low micromolar affinities (1966-1588 $\mathrm{nM}$ ) and the $\alpha 2$ receptor subtypes, in which all three PEA analogs showed affinities in the range of their transporter releasing potency (411-2320 nM). DEPEA showed activity at DAT and NET (108-124 nM), whereas AEPEA and MEPEA did not. AEPEA and DEPEA had low micromolar affinities to inhibit binding to the $\sigma 2$ site, whereas AEPEA and MEPEA had low to mid micromolar affinities to inhibit binding at the histamine $\mathrm{H} 1$ site. Amphetamine showed activity at the $\alpha$ receptor subtypes and also at the NET but, interestingly, not at the DAT. Although this latter finding seems counterintuitive, previous studies demonstrate that amphetamine displays weak ability to displace high-affinity phenyltropane analogs at monoamine transporters (Rothman et al., 1999; Eshleman et al., 1999, 2017).

Biotelemetry in Rats. Rats quickly adapted to the experimental injection procedure, and results for the control conditions remained relatively stable throughout testing.
Figure 4 depicts representative time course effects of saline or AEPEA injection on BP, HR, activity, and body temperature. In saline-treated rats, BP rapidly decreased and stabilized over the 3-hour session, whereas AEPEA produced time-related $\left(\mathrm{F}_{17,338}=14.9, P<0.0001\right)$ and dose-related $\left(\mathrm{F}_{4,20}=18.0, P<0.0001\right)$ elevations in $\mathrm{BP}$ that were maintained throughout the session. The hypertensive effect of AEPEA was significantly greater than saline at all doses (Dunnett's $P<0.05$ ). The HR response to AEPEA displayed significant effects of time $\left(\mathrm{F}_{17,338}=7.3, P<0.0001\right)$ but not dose $\left(\mathrm{F}_{4,20}=2.3, P=0.098\right)$, even though the higher doses of AEPEA caused modest tachycardia toward the end of the session. AEPEA produced small increases in motor activity at the beginning of the session, which were significant with respect to time $\left(\mathrm{F}_{17,338}=17.1, P<0.0001\right)$ but not dose $\left(\mathrm{F}_{4,20}=1.7, P=0.1905\right)$. Core body temperature was not significantly altered by AEPEA administration $\left(\mathrm{F}_{4,20}=1.7\right.$, $P=0.1922)$, despite a hypothermic response at the highest dose administered.

TABLE 2

G Protein-coupled receptorome screening for amphetamine and PEA analogs ${ }^{\mathrm{a}}$

Data represent $\mathrm{K}_{\mathrm{i}}$ (nanomolar) values obtained from nonlinear regression using the Cheng-Prusoff equation when inhibition was $>50 \%$. The results for amphetamine (AMPH) were reported previously (Schindler et al., 2019).

\begin{tabular}{|c|c|c|c|c|}
\hline & AMPH & AEPEA & MEPEA & DEPEA \\
\hline \multicolumn{5}{|l|}{ Serotonin } \\
\hline 5-HT1A & 2625 & $>10,000$ & 1966 & 1588 \\
\hline 5-HT2B & 971 & $>10,000$ & $>10,000$ & $>10,000$ \\
\hline$\alpha 2 \mathrm{~A}$ & 420 & $>10,000$ & $>10,000$ & $>10,000$ \\
\hline$\alpha 2 \mathrm{~B}$ & 192 & 1362 & 411 & 1013 \\
\hline$\alpha 2 \mathrm{C}$ & 171 & 1404 & 1805 & 2320 \\
\hline NET & 31 & $>10,000$ & $>10,000$ & 108 \\
\hline \multicolumn{5}{|l|}{ Sigma } \\
\hline$\sigma 2$ & $>10,000$ & $>10,000$ & 806 & 1352 \\
\hline \multicolumn{5}{|l|}{ Histamine } \\
\hline H1 & $>10,000$ & 2541 & 5800 & $>10,000$ \\
\hline
\end{tabular}

${ }^{a} \mathrm{~K}_{\mathrm{i}}$ values for the following receptors were $>10,000 \mathrm{nM}$ for all four drugs tested: serotonin receptors 5-HT1B, 5-HT1D, 5-HT1E, 5-HT2A, 5-HT2C, 5-HT3, 5-HT5A, 5-HT6, 5-

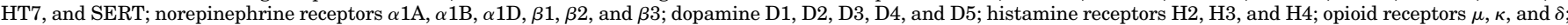
muscarinic receptors $\mathrm{M} 1, \mathrm{M} 2, \mathrm{M} 3, \mathrm{M} 4$, and M5; $\sigma 1$ receptor; benzodiazepine and peripheral benzodiazepine receptors. 

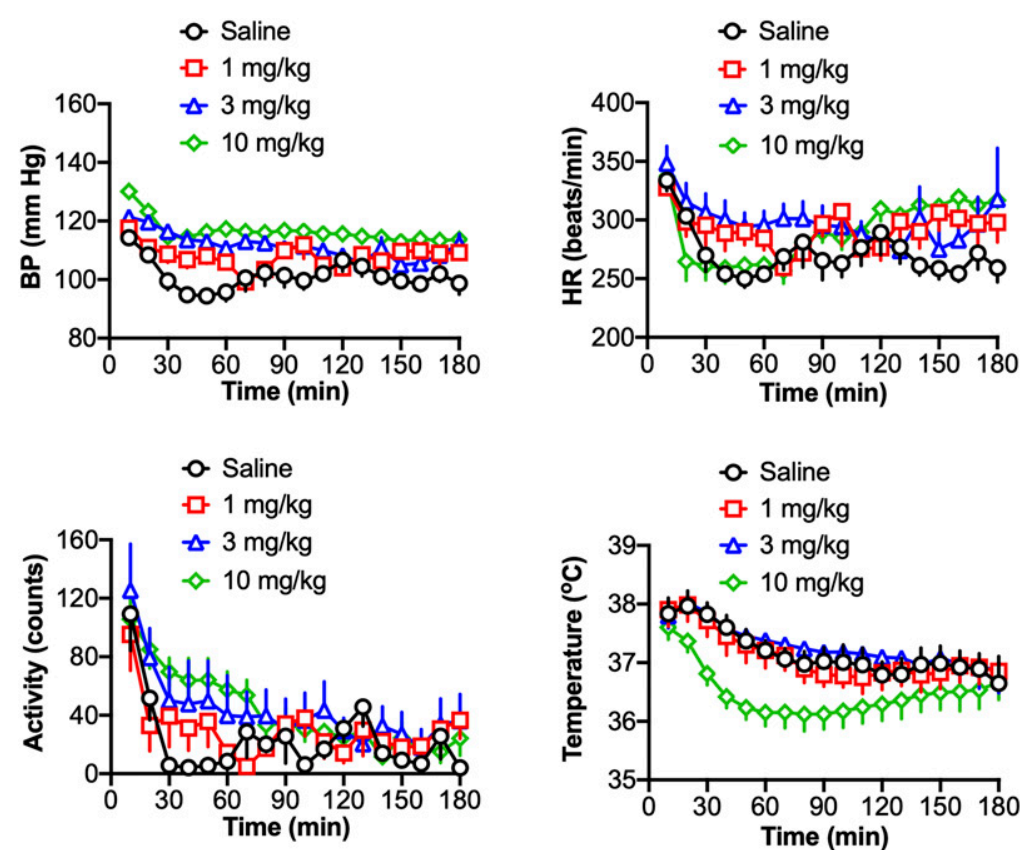

Fig. 4. Time course effects of AEPEA administration on BP, HR, motor activity, and core body temperature. Male rats bearing biotelemetry transponders received subcutaneous injection of 1,3 , or $10 \mathrm{mg} / \mathrm{kg}$ AEPEA or its saline vehicle and were returned to their home cages. Five minutes later, cages were placed atop telemetric receivers. Data were collected in 10minute epochs for 3 hours. Data are expressed as means \pm S.E.M. for five rats per group.
The time course data from Fig. 4 demonstrated that BP effects of AEPEA lasted for most of the 3-hour session, and the effects of MEPEA and DEPEA followed similar time courses (data not shown). Given this information, we examined doseresponse relationships for the averaged responses over the 3hour sessions. Figure 5 shows the effects of amphetamine and the PEA analogs on BP, HR, locomotor activity, and body temperature averaged over the full 3-hour session. As expected, amphetamine produced dose-dependent increases in $\mathrm{BP}\left(\mathrm{F}_{4,24}=27.7, P<0.0001\right)$ and $\mathrm{HR}\left(\mathrm{F}_{4,24}=9.4, P=0.0002\right)$, with the highest three doses $(0.3-3.0 \mathrm{mg} / \mathrm{kg})$ producing increases in $\mathrm{BP}$ and $\mathrm{HR}$ significantly above saline control.
Amphetamine also produced increases in locomotor activity $\left(\mathrm{F}_{4,24}=5.4, P=0.0041\right)$, although those effects were not dosedependent. Activity increased as dose increased up to $1 \mathrm{mg} / \mathrm{kg}$, which was significantly above saline levels. However, at the dose of $3 \mathrm{mg} / \mathrm{kg}$, activity decreased when compared with the effect of $1 \mathrm{mg} / \mathrm{kg}$. This apparent suppression of activity most likely reflects an increase in stereotypy that is not measured by the telemetry devices. At the doses tested, amphetamine did not significantly affect body temperature.

Similar to amphetamine, all three PEA analogs increased $\mathrm{BP}$ in a dose-dependent manner (AEPEA $\mathrm{F}_{4,24}=28.4, P<$ 0.0001 ; MEPEA F $\mathrm{F}_{3,19}=17.2, P<0.0001 ; \mathrm{DEPEA} \mathrm{F}_{3,19}=10.6$,
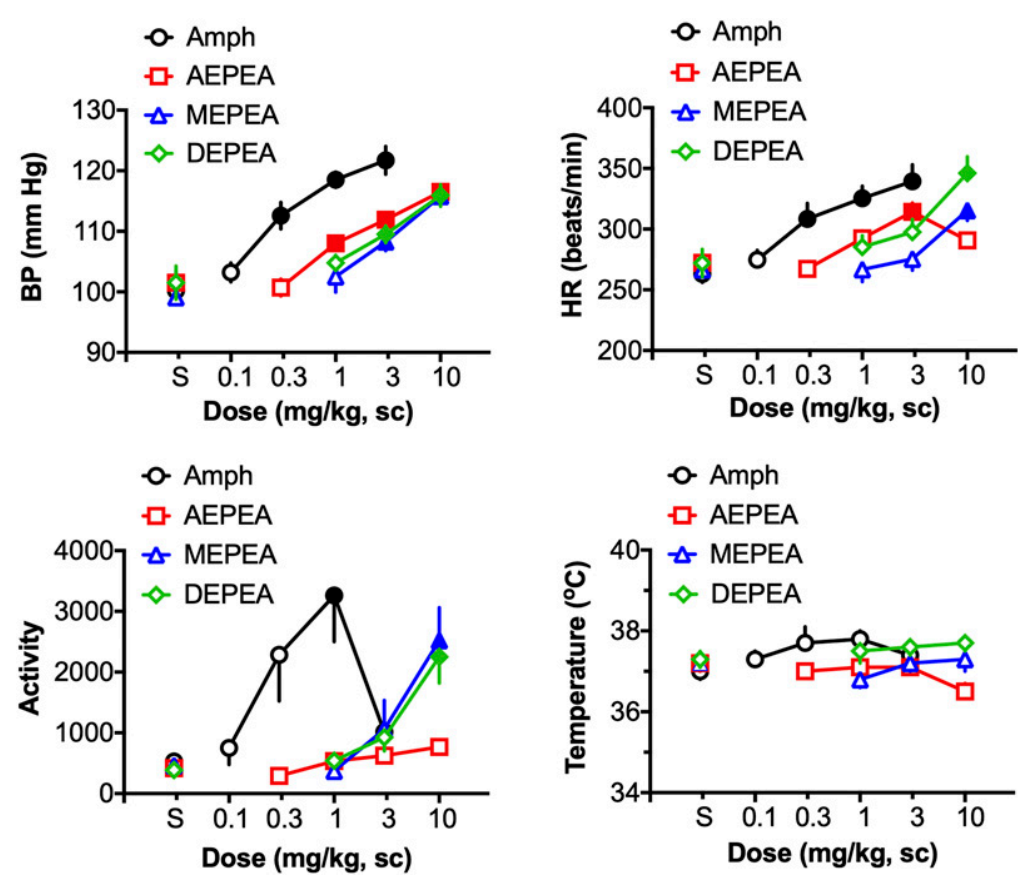

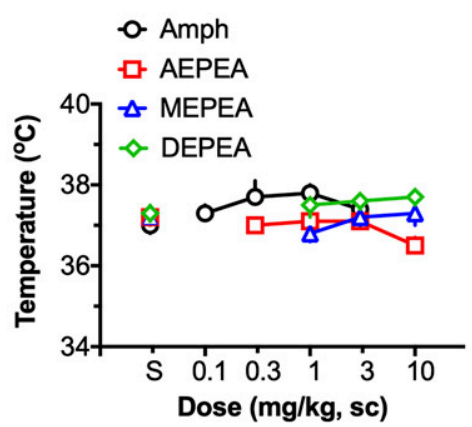

Fig. 5. Dose-effect functions for amphetamine (Amph) and PEA analogs on BP, HR, motor activity, and core body temperature. Data represent mean values across the full 3hour session. Solid symbols indicate significant differences from the respective saline group. Data are means \pm S.E.M. for five rats per group. 
$P=0.0004$ ), with higher doses of each drug producing increases in BP significantly above saline control. The maximal effects of each drug on BP were similar. When only the doses inducing maximal effects were compared by one-way ANOVA, the overall effect was significant $\left(\mathrm{F}_{3,16}=3.5, P=\right.$ 0.04); however, comparisons between the drugs (Tukey) failed to show any significant differences between any of the PEA analogs and amphetamine. MEPEA and DEPEA also produced dose-dependent increases in HR (MEPEA $F_{3,19}=6.6$, $P<0.01$; DEPEA $\mathrm{F}_{3,19}=8.1, P=0.0017$ ), with the effects of each drug at the highest dose tested being significantly above saline control. The effects of AEPEA $\left(\mathrm{F}_{4,24}=8.6, P=.0003\right)$ were more complicated, with HR increasing after treatment doses up to $3 \mathrm{mg} / \mathrm{kg}$ but then returning toward saline levels at $10 \mathrm{mg} / \mathrm{kg}$. For both their effects on BP and HR, the PEA analogs appeared to be approximately 10 times less potent than amphetamine. Like with BP, the maximal effects of each drug were similar $\left(\mathrm{F}_{3,16}=2.1, P=0.14\right)$.

Because all of the test drugs produced dose-dependent increases in $\mathrm{BP}, \mathrm{ED}_{50}$ values were calculated based on the maximal effect of amphetamine compared with saline. The calculated $\mathrm{ED}_{50}$ for amphetamine was $0.31 \mathrm{mg} / \mathrm{kg}(95 \% \mathrm{CI}=$ $0.17-0.48 \mathrm{mg} / \mathrm{kg}$ ). The $\mathrm{ED}_{50}$ values for the PEA analogs were from 8- to 14-fold greater than that of amphetamine, and the 95\% CIs for the PEA analogs did not overlap with those of amphetamine (AEPEA ED $50=2.54 \mathrm{mg} / \mathrm{kg}, 95 \% \mathrm{CI}=1.89$ $3.53 \mathrm{mg} / \mathrm{kg}$; DEPEA ED $50=3.74 \mathrm{mg} / \mathrm{kg}, \mathrm{CI}=2.33-6.71 \mathrm{mg} / \mathrm{kg}$; MEPEA ED ${ }_{50}=4.47 \mathrm{mg} / \mathrm{kg}, \mathrm{CI}=2.80-9.02 \mathrm{mg} / \mathrm{kg}$ ). Moreover, an $\mathrm{EC}_{50}$ shift analysis confirmed that the potency of amphetamine to induce increases in BP was significantly greater than the potencies of the PEA compounds $\left(\mathrm{F}_{3,56}=13.3, P<0.0001\right)$, although the effects of AEPEA, MEPEA, and DEPEA on BP did not differ from each other. Taken together, these results support the conclusion that the PEA analogs are generally 10 times less potent than amphetamine at increasing BP.

Similar to amphetamine, MEPEA and DEPEA produced significant increases in locomotor activity $\left(\mathrm{MEPEA} \mathrm{F}_{3,19}=7.4\right.$, $P=0.0025$; DEPEA $F_{3,19}=10.9, P=0.0004$ ), with the increases at $10 \mathrm{mg} / \mathrm{kg}$ being significantly above saline controls. In contrast to amphetamine, there was no downturn in activity at the highest doses of MEPEA or DEPEA, although doses above $10 \mathrm{mg} / \mathrm{kg}$ were not tested. For their effects on locomotor activity, MEPEA and DEPEA were at least 10 times less potent than amphetamine. There was a trend for AEPEA to also produce small dose-related increases in activity $\left(\mathrm{F}_{4,24}=\right.$ $3.1, P=0.0376$ ); however, follow-up tests failed to reveal any significant changes from saline control at any dose tested. Unlike with BP and HR, the maximal effects for the drugs on locomotor activity were different for the dose range tested. When the maximal effects were compared $\left(\mathrm{F}_{3,16}=4.1, P=\right.$ 0.02 ), the effects of AEPEA were significantly different from amphetamine. None of the PEA analogs produced significant effects on body temperature.

\section{Discussion}

PEA analogs continue to be found in dietary supplements, despite being banned by the FDA (Cohen et al., 2014; Elsohly et al., 2015; Zhao et al., 2018). Here, we studied compounds that possess an ethyl group at the $\alpha$-position carbon, in contrast to amphetamine, which has a methyl group at this position. Our results show that compounds found in nutritional supplements can have effects that mimic those produced by the abused psychostimulant amphetamine. Like amphetamine, all three of the structurally related PEAs produced statistically significant elevations in BP, and MEPEA and DEPEA also increased HR. MEPEA and DEPEA produced increases in locomotor activity similar to amphetamine. In general, the PEAs were about 10 times less potent than amphetamine. If supplement products are taken orally according to the manufacturer's recommendations, the amount of DEPEA ingested in these nutritional supplements would be 35-45 mg per serving (Cohen et al., 2014; ElSohly et al., 2015). In humans, the threshold oral dose of amphetamine that can be discriminated is $5 \mathrm{mg}$ (Chait et al., 1985), whereas amphetamine increases $\mathrm{BP}$ and $\mathrm{HR}$ at oral doses as low as 7.5-10 mg (Brauer and de Wit, 1996; Kelly et al., 2006; Harvanko et al., 2016). If the relative potency estimates for PEA analogs observed here for subcutaneous administration in rats hold for oral consumption in humans, a single serving of the supplement containing one of the compounds may be sufficient to produce similar subjective effects to amphetamine, and as little as two servings may produce cardiovascular effects. Thus, it is possible for an individual to use these supplements in amounts that could produce amphetaminelike effects, including mood stimulation and cardiovascular complications.

Although the PEAs all produced similar effects on BP with comparable potencies, there were substantial differences in their other in vivo effects. DEPEA appeared to produce larger and more potent effects on HR. MEPEA may produce larger HR effects at higher doses, but the effect of AEPEA appeared to peak at $3 \mathrm{mg} / \mathrm{kg}$. Unlike DEPEA and MEPEA, AEPEA had minimal effects on locomotor activity at the dose range tested. These differences in effects on activity may translate into differences for other behavioral effects, such as drug selfadministration, although additional research would be needed to confirm this. None of the drugs, including amphetamine, had significant effects on body temperature at the doses tested.

As expected, in the release assays, amphetamine was a potent efficacious releaser at both DAT and NET in rat brain synaptosomes (Rothman and Baumann, 2003). AEPEA and MEPEA were also releasers, but they were less potent than amphetamine and displayed selectivity for the NET over the DAT. The releasing abilities of AEPEA and MEPEA were antagonized by inhibitors of DAT and NET, implicating monoamine transporters in their effects. However, any definitive conclusions about the molecular mechanism of action for PEA compounds will require the replication of our findings using transfected cells expressing pure populations of DAT and NET. Despite the similarity of effects produced by AEPEA and MEPEA in the in vitro assays, MEPEA significantly increased locomotor activity in vivo, whereas AEPEA did not. DEPEA displayed fully efficacious release at NET but only weak partial releasing activity at DAT (i.e., $40 \%$ of $\mathrm{E}_{\max }$ ). The precise molecular underpinnings of DEPEA's partial releasing activity at DAT is not known, but we speculate that uptake inhibition at DAT is the predominant effect of DEPEA at this transporter. Like the other PEA analogs, DEPEA was slightly more potent at NET than DAT. In general, DEPEA was less potent than AEPEA or MEPEA at both DAT and NET in vitro but was at least equipotent to both MEPEA and AEPEA on $\mathrm{BP}$ and $\mathrm{HR}$ in vivo and clearly more potent than AEPEA on 
locomotor activity. This pattern of results suggests that in vivo effects of these PEA analogs are influenced by factors other than uptake inhibition or release properties at DAT and NET, such as pharmacokinetic factors or actions at other binding sites.

The $\alpha$-ethyl-substituted PEA analogs failed to interact (i.e., $\mathrm{K}_{\mathrm{i}}>10 \mu \mathrm{M}$ ) with most of the GPCRs studied (footnote of Table 2). Further, for the few receptors they bind to, the compounds do not show potency relationships that mirrored any of the in vivo effects observed. For example, at the 5-HT1A receptor, amphetamine was equipotent to MEPEA and DEPEA, but amphetamine was much more potent than both of these compounds in all measures of in vivo activity. At the $\alpha 2 \mathrm{~B}$ receptor, MEPEA appears to have twice the potency of DEPEA; however, MEPEA displayed equivalent or less potency than DEPEA for the in vivo tests. Therefore, it does not appear as though activity at these other receptors is responsible for the observed in vivo effects. Given the fact that amphetamine showed clearly greater potency than the PEA analogs on the DAT and NET release assays and that the same potency relationship held for the in vivo tests, actions at DAT and NET seem most likely to mediate the in vivo effects, although we cannot rule out that pharmacokinetic effects may be responsible for some of the observed differences.

In a previous study (Schindler et al., 2019), we examined the pharmacological effects of a PEA analog in which the $\alpha$-methyl group of amphetamine was moved to the $\beta$-position to form BMPEA. This change reduced the potency of BMPEA to increase $\mathrm{BP}$ about 30-fold when compared with amphetamine, and at the doses tested, BMPEA did not increase HR or activity. BMPEA maintained substrate activity at DAT and NET but was more potent at NET than DAT. By comparison, in the experiments reported here, replacing the $\alpha$-methyl group of amphetamine and methamphetamine with an $\alpha$-ethyl group to yield AEPEA and MEPEA led to a reduction in potency of only about 10-fold for the in vivo effects and also maintained releaser efficacy. Importantly, the $\alpha$-ethyl compounds seem to show a preference for the NET over the DAT, which is similar to the profile of BMPEA. Replacing the amine methyl group of MEPEA with an ethyl group to produce DEPEA markedly reduced the efficacy of release at DAT, and this observation agrees with findings reported for amphetamine analogs with extended $N$-alkyl chain length (see Solis et al., 2017). Overall, replacing the $\alpha$-methyl group of amphetamine with an ethyl group maintains the amphetamine-like effects of PEA analogs more effectively than moving the methyl group to the $\beta$-position for BMPEA. Thus, $\alpha$-ethyl-substituted analogs may also have abuse potential similar to amphetamine.

Although not directly studied here, it is likely that locomotoractivating effects of $\alpha$-ethyl compounds are related to their effects at the DAT. Activity at DAT is known to be primarily responsible for the locomotor-activating effects of a variety of stimulants, including cocaine, amphetamine, and related analogs (Uhl et al., 2002; Rothman and Baumann, 2003; Zolkowska et al., 2009). Activity at DAT is also known to be primarily responsible for maintaining drug self-administration (Wise and Bozarth, 1985; Uhl et al., 2002; Howell and Kimmel, 2008), and our present findings suggest that at least some of the $\alpha$-ethyl-substituted analogs will be self-administered and have abuse potential in humans. Likewise, previous work with other psychomotor stimulants has shown that central or peripheral noradrenergic systems are primarily responsible for their cardiovascular effects. For example, increases in BP produced by BMPEA were blocked by the $\alpha$-adrenergic antagonist prazosin (Schindler et al., 2019). $\beta$-Adrenergic antagonists can block the HR-increasing effects of psychomotor stimulants, including some amphetamines (Schindler et al., 1992b, 2014), cathinones (Varner et al., 2013; Alsufyani and Docherty, 2015; Schindler et al., 2016), and cocaine (Branch and Knuepfer, 1992; Schindler et al., 1992a). With respect to cocaine, although pretreatment with $\beta$ antagonists can antagonize tachycardia, some studies show that such antagonists can exacerbate the hypertensive effects (Branch and Knuepfer, 1992; Schindler et al., 1992a).

Limitations of the current study include the measurement of only $\mathrm{BP}$ and $\mathrm{HR}$ as indices of cardiac function. Although increases in both $\mathrm{BP}$ and $\mathrm{HR}$ would be expected to increase the workload on the heart and lead to potential adverse effects, the assessment of other relevant endpoints, such as cardiac output, cardiac electrical activity, and contractility (Mladěnka et al., 2018), would produce a more complete picture of the effects of $\alpha$-ethyl-substituted PEAs on cardiac function. Nevertheless, the effects shown here do raise concern for the presence of these substances in dietary supplements, particularly if they are not listed on the ingredients label. In addition, the in vivo effects reported here were observed after passive administration of the compounds. In humans, these substances would be self-administered, which could influence the effects of the compounds on cardiac function, as well as other measures (Hemby et al., 1997; Graziella De Montis et al., 1998).

In summary, similar to amphetamine, AEPEA and MEPEA function as efficacious neurotransmitter releasers at DAT and NET, but with greater potency at NET relative to DAT. By contrast, DEPEA displays efficacious release at NET but lowefficacy partial release at DAT. Despite these minor differences from amphetamine in neurochemical mechanism, all three $\alpha$-ethyl PEA analogs increased BP similar to amphetamine, although at reduced potency. MEPEA and DEPEA also increased HR. As a result, these compounds could produce toxic effects if taken in large enough amounts. Like amphetamine, MEPEA and DEPEA increased locomotor activity, suggesting these agents could have central effects, including abuse potential in humans.

\section{Authorship Contributions}

Participated in research design: Schindler, Baumann.

Conducted experiments: Schindler, Thorndike, Partilla, Baumann. Contributed new reagents or analytic tools: Rice.

Performed data analysis: Schindler, Thorndike, Baumann.

Wrote or contributed to writing of the manuscript: Schindler, Partilla, Rice, Baumann.

\section{References}

Alsufyani HA and Docherty JR (2015) Direct and indirect cardiovascular actions of cathinone and MDMA in the anaesthetized rat. Eur J Pharmacol 758:142-146. Baumann MH, Partilla JS, Lehner KR, Thorndike EB, Hoffman AF, Holy M, Rothman RB, Goldberg SR, Lupica CR, Sitte HH, et al. (2013) Powerful cocaine-like actions of 3,4-methylenedioxypyrovalerone (MDPV), a principal constituent of psychoactive 'bath salts' products. Neuropsychopharmacology 38:552-562.

Besnard J, Ruda GF, Setola V, Abecassis K, Rodriguiz RM, Huang XP, Norval S, Sassano MF, Shin AI, Webster LA, et al. (2012) Automated design of ligands to polypharmacological profiles. Nature 492:215-220.

Branch CA and Knuepfer MM (1992) Adrenergic mechanisms underlying cardiac and vascular responses to cocaine in conscious rats. J Pharmacol Exp Ther 263: $742-751$.

Brauer LH and de Wit H (1996) Subjective responses to d-amphetamine alone and after pimozide pretreatment in normal, healthy volunteers. Biol Psychiatry 39: $26-32$. 
Chait LD, Uhlenhuth EH, and Johanson CE (1985) The discriminative stimulus and subjective effects of d-amphetamine in humans. Psychopharmacology (Berl) 86: 307-312.

Cohen PA, Travis JC, and Venhuis BJ (2014) A methamphetamine analog (N, $\alpha-$ diethyl-phenylethylamine) identified in a mainstream dietary supplement. Drug Test Anal 6:805-807.

Dreher M, Ehlert T, Simon P, and Neuberger EWI (2018) Boost me: prevalence and reasons for the use of stimulant containing pre workout supplements among fitness studio visitors in Mainz (Germany). Front Psychol 9:1134.

Eichner ER (2014) Fatal caffeine overdose and other risks from dietary supplements. Curr Sports Med Rep 13:353-354.

ElSohly MA and Gul W (2014) LC-MS-MS analysis of dietary supplements for N-ethyl- $\alpha$-ethyl-phenethylamine (ETH), N, N-diethylphenethylamine and phenethylamine. J Anal Toxicol 38:63-72.

ElSohly MA, Murphy TP, ElSohly KM, and Gul W (2015) LC-MS-MS analysis of N, $\alpha$ Diethylphenethylamine $(\mathrm{N}, \alpha-\mathrm{ETH})$ and its positional isomer $\mathrm{N}, \beta$-Diethylphenethylamine (N, $\beta-E T H)$ in dietary supplements. J Anal Toxicol 39:387-406.

Eshleman AJ, Carmolli M, Cumbay M, Martens CR, Neve KA, and Janowsky A (1999) Characteristics of drug interactions with recombinant biogenic amine transporters expressed in the same cell type. J Pharmacol Exp Ther 289:877-885.

Eshleman AJ, Wolfrum KM, Reed JF, Kim SO, Swanson T, Johnson RA and Janowsky A (2017) Structure-activity relationships of substituted cathinones, with transporter binding, uptake, and release. J Pharmacol Exp Ther 360:33-47.

González-Sabin J, Gotor V, and Rebolledo F (2002) CAL-B-Catalyzed resolution of some pharmacologically interesting $\beta$-substituted isopropylamines. Tetrahedron Asymmetry 13:1315-1320.

Graziella De Montis M, Co C, Dworkin SI, and Smith JE (1998) Modifications of dopamine D1 receptor complex in rats self-administering cocaine. Eur J Pharmaco 362:9-15.

Harvanko A, Martin C, Lile J, Kryscio R, and Kelly TH (2016) Individual differences in the reinforcing and subjective effects of d-amphetamine: dimensions of impulsivity. Exp Clin Psychopharmacol 24:436-446.

Hemby SE, Co C, Koves TR, Smith JE, and Dworkin SI (1997) Differences in extracellular dopamine concentrations in the nucleus accumbens during responsedependent and response-independent cocaine administration in the rat. Psychopharmacology (Berl) 133:7-16.

Howell LL and Kimmel HL (2008) Monoamine transporters and psychostimulant addiction. Biochem Pharmacol 75:196-217.

Kelly TH, Robbins G, Martin CA, Fillmore MT, Lane SD, Harrington NG, and Rush CR (2006) Individual differences in drug abuse vulnerability: d-amphetamine and sensation-seeking status. Psychopharmacology (Berl) 189:17-25.

Lee j, Choe S, Choi H, Heo S, Kim E, Kim H, Bang E, and Chung H (2013) Identification of N-ethyl- $\alpha$-ethylphenethylamine in crystalline powder seized for suspected drug trafficking: a reserach chemical or a new designer drug? Forensic Toxicol 31:54-58.

Liu Y and Santillo MF (2016) Cytochrome P450 2D6 and 3A4 enzyme inhibition by amine stimulants in dietary supplements. Drug Test Anal 8:307-310.

Mladěnka P, Applová L, Patočka J, Costa VM, Remiao F, Pourová J, Mladěnka A, Karlíčková J, Jahodár L, Vopršalová M, et al.; TOX-OER and CARDIOTOX Hradec Králové Researchers and Collaborators (2018) Comprehensive review of cardiovascular toxicity of drugs and related agents. Med Res Rev 38: 1332-1403.

Oberlender R and Nichols DE (1991) Structural variation and (+)-amphetamine-like discriminative stimulus properties. Pharmacol Biochem Behav 38:581-586.

Pawar RS and Grundel E (2017) Overview of regulation of dietary supplements in the USA and issues of adulteration with phenethylamines (PEAs). Drug Test Anal 9 $500-517$.

Rothman RB and Baumann MH (2003) Monoamine transporters and psychostimulant drugs. Eur J Pharmacol 479:23-40.

Rothman RB, Baumann MH, Dersch CM, Appel J, and Houghten RA (1999) Discovery of novel peptidic dopamine transporter ligands by screening a positional scanning combinatorial hexapeptide library. Synapse 33:239-246.
Rothman RB, Clark RD, Partilla JS, and Baumann MH (2003) (+)-Fenfluramine and its major metabolite, (+)-norfenfluramine, are potent substrates for norepinephrine transporters. J Pharmacol Exp Ther 305:1191-1199.

Santillo MF (2014) Inhibition of monoamine oxidase (MAO) by $\alpha$-ethylphenethylamine and $\mathrm{N}, \alpha$-diethylphenethylamine, two compounds related to dietary supplements. Food Chem Toxicol 74:265-269.

Schindler CW, Tella SR, and Goldberg SR (1992a) Adrenoceptor mechanisms in the cardiovascular effects of cocaine in conscious squirrel monkeys. Life Sci 51: $653-660$.

Schindler CW, Thorndike EB, Blough BE, Tella SR, Goldberg SR, and Baumann MH (2014) Effects of 3,4-methylenedioxymethamphetamine (MDMA) and its main metabolites on cardiovascular function in conscious rats. $\mathrm{Br} J$ Pharmacol 171: 83-91.

Schindler CW, Thorndike EB, Rice KC, Partilla JS, and Baumann MH (2019) The supplement adulterant $\beta$-methylphenethylamine increases blood pressure by acting at peripheral norepinephrine transporters. J Pharmacol Exp Ther 369: $328-336$.

Schindler CW, Thorndike EB, Suzuki M, Rice KC, and Baumann MH (2016) Pharmacological mechanisms underlying the cardiovascular effects of the "bath salt" constituent 3,4-methylenedioxypyrovalerone (MDPV). $\mathrm{Br} J$ Pharmacol 173: $3492-3501$

Schindler CW, Zheng JW, Tella SR, and Goldberg SR (1992b) Pharmacological mechanisms in the cardiovascular effects of methamphetamine in conscious squirrel monkeys. Pharmacol Biochem Behav 42:791-796.

Solis E Jr, Partilla JS, Sakloth F, Ruchala I, Schwienteck KL, De Felice LJ, Eltit JM, Glennon RA, Negus SS, and Baumann MH (2017) N-alkylated analogs of 4 methylamphetamine (4-MA) differentially affect monoamine transporters and abuse liability. Neuropsychopharmacology 42:1950-1961.

Uhl GR, Hall FS, and Sora I (2002) Cocaine, reward, movement and monoamine transporters. Mol Psychiatry 7:21-26.

Uralets V, App M, Rana S, Morgan S, and Ross W (2014) Designer phenethylamines routinely found in human urine: 2-ethylamino-1-phenylbutane and 2-amino-1phenylbutane. J Anal Toxicol 38:106-109.

Varner KJ, Daigle K Weed PF, Lewis PB, Mahne SE, Sankaranarayanan A, and Winsauer PJ (2013) Comparison of the behavioral and cardiovascular effects of mephedrone with other drugs of abuse in rats. Psychopharmacology (Berl) 225: $675-685$.

Wahlstrom R, Styules C, and Hägglund G (2014) Reliable identification and quantification of three diethylphenethylamines in Dendrobium-based dietary supplement. Anal Methods 6:7891-7897.

Wise RA and Bozarth MA (1985) Brain mechanisms of drug reward and euphoria Psychiatr Med 3:445-460.

Wójtowicz M, Jarek A, Chajewska K, Turek-Lepa E, and Kwiatkowska D (2015) Determination of designer doping agent--2-ethylamino-1-phenylbutane--in dietary supplements and excretion study following single oral supplement dose. J Pharm Biomed Anal 115:523-533.

World Anti-Doping Agency (2012) 2012 Anti-doping Testing Figures Report, World Anti-Doping Agency, Montreal, Canada.

World Anti-Doping Agency (2013) 2013 Anti-doping Testing Figures Report, World Anti-Doping Agency, Montreal, Canada.

Zhao J, Wang M, Avula B, and Khan IA (2018) Detection and quantification of phenethylamines in sports dietary supplements by NMR approach. J Pharm Biomed Anal 151:347-355.

Zolkowska D, Jain R, Rothman RB, Partilla JS, Roth BL, Setola V, Prisinzano TE, and Baumann MH (2009) Evidence for the involvement of dopamine transporters in behavioral stimulant effects of modafinil. J Pharmacol Exp Ther 329:738-746.

Address correspondence to: Charles W. Schindler, Designer Drug Research Unit, IRP, NIDA, NIH, DHHS, 251 Bayview Blvd., Baltimore, MD 21224. E-mail: cschind@helix.nih.gov 